\title{
A Quantum Implementation Model for Artificial Neural Networks
}

\author{
Ammar Daskin \\ Computer Engineering Department, Istanbul Medeniyet University, Istanbul, Turkey. E-mail: adaskin25@gmail.com
}

Editors: George Svetlichny, Danko Georgiev \& Chris C. King

Article history: Submitted on October 12, 2017; Accepted on January 30, 2018; Published on February 20, 2018.

( he learning process for multilayered neural networks with many nodes makes heavy demands on computational resources. In some neural network models, the learning formulas, such as the Widrow-Hoff formula, do not change the eigenvectors of the weight matrix while flatting the eigenvalues. In infinity, these iterative formulas result in terms formed by the principal components of the weight matrix, namely, the eigenvectors corresponding to the non-zero eigenvalues. In quantum computing, the phase estimation algorithm is known to provide speedups over the conventional algorithms for the eigenvalue-related problems. Combining the quantum amplitude amplification with the phase estimation algorithm, a quantum implementation model for artificial neural networks using the Widrow-Hoff learning rule is presented. The complexity of the model is found to be linear in the size of the weight matrix. This provides a quadratic improvement over the classical algorithms.

Quanta 2018; 7: 7-18.

\section{Introduction}

Artificial neural networks [1-3] are adaptive statistical models which mimic the neural structure of the human brain to find optimal solutions for multivariate problems. In the design of artificial neural networks are determined the following: the structure of the network, input-output variables, local activation rules, and a learning algorithm. Learning algorithms are generally linked to the activities of neurons and describe a mathematical cost function. Often, a minimization of this cost function composed of the weights and biases describes the learning process in artificial neural networks. Moreover, the learning rule in this process specifies how the synaptic weights should be updated at each iteration. In general, learning rules can be categorized as supervised and unsupervised learning: In supervised learning rules, the distance between the response of the neuron and a specified response, called target $t$, is considered. However, it is not required in unsupervised learning rules. Hebbian learning rule [4] is a typical example of the unsupervised learning, in which the weight vector at the $(j+1)$ th iteration is updated by the following formula (we will mainly follow [2] to describe learning rules):

$$
\mathbf{w}_{[\mathbf{j}+\mathbf{1}]}=\mathbf{w}_{[\mathbf{j}]}-\eta t \mathbf{x} .
$$

Here, $\mathbf{x}$ is the input vector, $\eta$ is a positive learning constant and $\mathbf{w}_{[\mathbf{j}]}$ represents the weights at the $j$ th iteration. And $t$ is the target response. Learning is defined by getting an output closer to the target response. 
On the other hand, Widrow-Hoff learning rule [5], which is the main interest of this paper, illustrates a typical supervised learning rule [2, 3, 6]

$$
\mathbf{w}_{[\mathbf{j}+\mathbf{1}]}=\mathbf{w}_{[\mathbf{j}]}-\eta \sigma^{\prime}(v)(t-y) \mathbf{x},
$$

where $v=\mathbf{x}^{T} \mathbf{w}$ is the activation of the output cell and $\sigma^{\prime}(v)$ is the derivative of the activation function which specifies the output of a cell in the considered network, $y=\sigma(v)$ : e.g., the sigmoid function, $\sigma(v)=$ $1 /(1+\exp (-v))$. While in the Hebbian iteration the weight vector is moved in the direction of the input vector by an amount proportional to the target, in the Widrow-Hoff iteration, the change is proportional to the error $(t-y)$. If we consider multi-neurons; the activation, the output, and the target values becomes vectors: viz., $\mathbf{v}, \mathbf{y}$ and $\mathbf{t}$, respectively. When there are several input and target associations, the set of inputs, targets, activations, and outputs can be represented by the matrices $X, T, V$, and $Y$, respectively. Then, the above equations come in matrix forms as follows

$$
\begin{gathered}
W_{[j+1]}=W_{[j]}-\eta X T^{T}, \\
W_{[j+1]}=W_{[j]}-\eta\left(\sigma^{\prime}(V) \circledast X\right)(T-Y)^{T},
\end{gathered}
$$

where $W$ represents the matrix of synaptic weights.

It is known that the learning task for multilayered neural networks with many nodes makes heavy demands on computational resources. Algorithms in quantum computational model provide computational speedup over their classical counterparts for some particular problems: e.g., Shor's factoring algorithm [7] and Grover's search algorithm [8]. Using adiabatic quantum computation [9, 10] or mapping data set to quantum random access memory [11 12] speedups in big data analysis have been shown to be possible [13-15]. Furthermore, Lloyd and collaborators [16] have described a quantum version for principal component analysis.

In the recent decades, relating the neurons in the networks with qubits [17], a few different quantum networks analogous of the artificial neural networks have also been developed: e.g. [18-23] (For a complete review and list of references, see [24]). These models should not be confused with the classical algorithms (cf. [25, 26]) inspired by the quantum computing. Furthermore, using the Grover search algorithm [8], a quantum associative memory is introduced [27]. Despite some promising results, there is still need for further research on new models [24].

The quantum phase estimation algorithm (PEA) [28] provides computational speedups over the known classical algorithms in eigenvalue related problems. The algorithm mainly finds the phase value of the eigenvalue of a unitary matrix (considered as the time evolution operator of a quantum Hamiltonian) for a given approximate eigenvector. Because of this property, PEA is ubiquitously used as a subcomponent of other algorithms. While in the general case, PEA requires a good initial estimate of an eigenvector to produce the phase; in some cases, it is able to find the phase by using an initial equal superposition state: e.g., Shor's factoring algorithm [7]. In [29], it is shown that a flag register can be used in the phase estimation algorithm to eliminate the ill-conditioned part of a matrix by processing the eigenvalues greater than some threshold value. Amplitude amplification algorithm [8, 30-32] is used to amplify amplitudes of certain chosen quantum states considered. In the definition of quantum reinforcement learning [33, 34], states and actions are represented as quantum states. And based on the observation of states a reward is applied to the register representing actions. Later, the quantum amplitude amplification is applied to amplify the amplitudes of rewarded states. In addition, in a prior work [35] combining the amplitude amplification with the phase estimation algorithm, we have showed a framework to obtain the eigenvalues in a given interval and their corresponding eigenvectors from an initial equal superposition state. This framework can be used as a way of doing quantum principal component analysis (QPCA).

For a given weight matrix $W$; in linear auto-associators using the Widrow-Hoff learning rule; during the learning process, the eigenvectors do not change while the eigenvalues go to one [2, 6]: i.e., $\lim _{j \rightarrow \infty} W_{[j]}$ converges to $Q Q^{T}$, where $Q$ represents the eigenvectors of $W$. Therefore, for a given input $\mathbf{x}$, the considered network produces the output $Q Q^{T} \mathbf{x}$. In this paper, we present a quantum implementation model for the artificial neural networks by employing the algorithm in [35]. In particular, we show how to construct $Q Q^{T} \mathbf{x}$ on quantum computers in linear time. In the following section, we give the necessary description of Widrow-Hoff learning rule and QPCA described in [35]. In Section 3, we shall show how to apply QPCA to the neural networks given by the Widrow-Hoff learning rule and discuss the possible implementation issues such as the circuit implementation of $W$, the preparation of the input $\mathbf{x}$ as a quantum circuit, and determining the number of iterations in the algorithm. In Section 4, we analyze the complexity of the whole application. Finally, in Section 5 , an illustrative example is presented.

\section{Methods}

In this section, we shall describe the Widrow-Hoff learning rule and the quantum algorithms used in the paper.

\subsection{Widrow-Hoff Learning}

For a linear autoassociator, i.e. $Y=V, \sigma^{\prime}(V)=I$, and $T=X$; Widrow-Hoff learning rule given in Eq. (4), 
also known as LMS algorithm, in matrix form can be described as follows [2,3]

$$
W_{[j]}=W_{[j-1]}+\eta\left(X-W_{[j-1]} X\right) X^{T} .
$$

This can be also expressed by using the eigendecomposition of $W=Q \Lambda Q^{T}$ : i.e., $W_{[j]}=Q \Phi_{[j]} Q^{T}$, where $\Phi_{[j]}=\left[I-(I-\eta \Lambda)^{j}\right] . \Phi_{[j]}$ is called the eigenvalue matrix at the epoch $j$. Based on this formulation, Widrow-Hoff error correction rule only affects the eigenvalues and flattens them when $\eta \leq 2 \lambda_{\max }^{-1}\left(\lambda_{\max }\right.$ is the largest eigenvalue of $W$ ): i.e., $\lim _{j \rightarrow \infty} \Phi_{[j]}=I$. Thus, in infinity, the learning process $W$ ends up as: $W_{[\infty]}=Q Q^{T}$.

\subsection{Quantum Algorithms Used in the Model}

In the following, we shall first explain two well-known quantum algorithms and then describe how they are used in [35] to obtain the linear combination of the eigenvectors.

\subsubsection{Quantum Phase Estimation Algorithm}

The phase estimation algorithm (PEA) finds an estimation for the phase of an eigenvalue of a given operator [28, 36]. In mathematical terms, the algorithm seen in Figure 1 as a circuit works as follows:

- An estimated eigenvector $\left|\varphi_{j}\right\rangle$ associated to the $j$ th eigenvalue $e^{\imath \phi_{j}}$ of a unitary matrix, $U$ of order $N$ is assumed given. $U$ is considered as a time evolution operator of the Hamiltonian $(H)$ representing the dynamics of the quantum system

$$
U=e^{l t H / \hbar},
$$

where $t$ represents the time and $\hbar$ is the Planck constant. As a result, the eigenvalues of $U$ and $H$ are related: while $e^{l \phi_{j}}$ is the eigenvalue of $U$, its phase $\phi_{j}$ is the eigenvalue of $H$.

- The algorithm uses two quantum registers dedicated to the eigenvalue and the eigenvector, respectively, $\left|r e g_{1}\right\rangle$ and $\left|r e g_{2}\right\rangle$ with $m$ and $\left(n=\log _{2} N\right)$ number of qubits. The initial state of the system is set to $\left|r e g_{1}\right\rangle\left|r e g_{2}\right\rangle=|\mathbf{0}\rangle\left|\varphi_{j}\right\rangle$, where $|\mathbf{0}\rangle$ is the first standard basis vector.

- Then, the quantum Fourier transform is applied to $\left|r e g_{1}\right\rangle$, which produces the following equal superposition state

$$
U_{Q F T}\left|r e g_{1}\right\rangle\left|r e g_{2}\right\rangle=\frac{1}{\sqrt{M}} \sum_{k=0}^{M-1}|\mathbf{k}\rangle\left|\varphi_{j}\right\rangle,
$$

where $M=2^{m}$ and $|\mathbf{k}\rangle$ is the $k$ th standard basis vector.

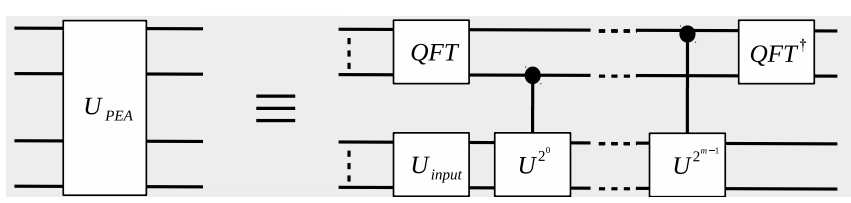

Figure 1: The phase estimation part of the algorithm.

- For each $k$ th qubit in the first register, a quantum operator, $U^{2^{k-1}}$, controlled by this qubit is applied to the second register. This operation leads the first register to hold the discrete Fourier transform of the phase, $\phi_{j}$.

- The inverse quantum Fourier transform on the first register produces the binary digits of $\phi_{j}$.

- Finally, the phase is obtained by measuring the first register.

\subsubsection{Quantum Amplitude Amplification Algorithm}

If a given quantum state $|\psi\rangle$ in $N$-dimensional Hilbert space can be rewritten in terms of some orthonormal states considered as the good and the bad parts of $|\psi\rangle$ as

$$
|\psi\rangle=\sin (\theta)\left|\psi_{\text {good }}\right\rangle+\cos (\theta)\left|\psi_{\text {bad }}\right\rangle,
$$

then amplitude amplification technique [8,32,37] can be used to increase the amplitude of $\left|\psi_{\text {good }}\right\rangle$ in magnitude while decreasing the amplitude of $\left|\psi_{b a d}\right\rangle$. The technique mainly consists of two parts: the marking and the amplifying implemented by two operators, respectively $U_{f}$ and $U_{\psi}$. Here, $U_{f}$ marks-flips the sign of-the amplitudes of $\left|\psi_{\text {good }}\right\rangle$ and does nothing to $\left|\psi_{\text {bad }}\right\rangle$. $U_{f}$ can be implemented as a reflection operator when $\left|\psi_{\text {good }}\right\rangle$ and $\left|\psi_{\text {bad }}\right\rangle$ are known

$$
U_{f}=I-2\left|\psi_{\text {good }}\right\rangle\left\langle\psi_{\text {good }}\right|,
$$

where $I$ is an identity matrix. In the amplification part, the marked amplitudes are amplified by the application of the operator $U_{\psi}$

$$
U_{\psi}=I-2|\psi\rangle\langle\psi|
$$

To maximize the probability of $\left|\psi_{\text {good }}\right\rangle$, the iteration operator $G=U_{\psi} U_{f}$ is applied iteratively $O(\sqrt{N})$ times to the resulting state.

\subsection{Quantum principal component analysis}

In [35], we have shown that combining PEA with the amplitude amplification, one can obtain eigenvalues in certain intervals. 
In the phase estimation part, the initial state of the registers is set to $|\mathbf{0}\rangle|\mathbf{0}\rangle$. Then, the second register is put into the equal superposition state $1 / \sqrt{N}(1, \ldots, 1)^{T}$. The phase estimation process in this input generates the superposition of the eigenvalues on the first and the eigenvectors on the second register. In this final superposition state, the amplitudes for the eigenpairs are proportional to the norm of the projection of the input vector onto the eigenvector: i.e., the normalized sum of the eigenvector elements. This part is represented by $U_{P E A}$ and also involves the input preparation circuit, $U_{\text {input }}$, on the second register.

In the amplification part, first, $U_{f}$ is applied to the first register to mark the eigenvalues determined by the binary values of the eigenvalues: For instance, if we want to mark an eigenvalue equal to 0.25 in $\left|r e g_{1}\right\rangle$ with 3 qubits, we use $U_{f}=I-2|010\rangle\langle 010|$ since the binary form of 0.25 is (010) (the left most bit represents the most significant bit). The amplitudes of the marked eigenvalues are then amplified by the application of $U_{\psi}$ with $|\psi\rangle$ representing the output of the phase estimation

$$
|\psi\rangle=U_{P E A}\left|r e g_{1}\right\rangle\left|r e g_{2}\right\rangle=U_{P E A}|\mathbf{0}\rangle|\mathbf{0}\rangle .
$$

Using the above equation, $U_{\psi}$ can be implemented as

$$
U_{\psi}=I-2|\psi\rangle\langle\psi|=U_{P E A} U_{0} U_{P E A}^{\dagger},
$$

where $U_{0}=I-2|\mathbf{0}\rangle\langle\mathbf{0}|$. The amplitudes of the eigenvalues in the desired region are further amplified by the iterative application of the operator $G=U_{\psi} U_{f}$. At the end of this process, a linear combination of the eigenvectors with the coefficients determined by the normalized sum of the vector elements of the eigenvectors are produced. In the following section, we shall show how to apply this process to model the implementation of the neural networks based on the Widrow-Hoff learning rule.

\section{Application to the Neural Networks}

Since the weight matrix in Widrow-Hoff learning rule converges to the principal components in infinity [6]: i.e., $W_{[\infty]}=Q Q^{T}$, the behavior of the trained network on some input $|\mathbf{x}\rangle$ can be concluded as

$$
W_{[\infty]}|\mathbf{x}\rangle=Q Q^{T}|\mathbf{x}\rangle .
$$

Our main purpose is to find an efficient way to implement this behavior on quantum computers by using the quantum principal component analysis. For this purpose, we form $U_{f}$ in a way that marks only the non-zero eigenvalues and their corresponding eigenvectors: For zero eigenvalues ( in binary form $(0 \ldots 0)$ ), the first register

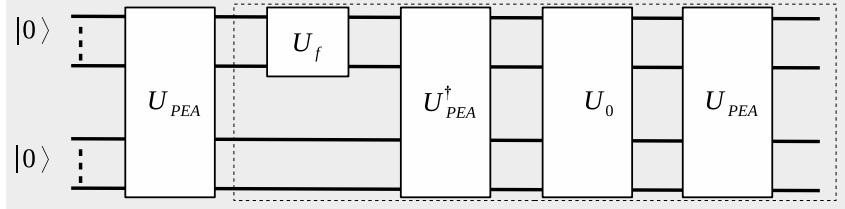

Figure 2: The general quantum algorithm to find the principal components of $W$. The dashed box indicates an iteration of the amplitude amplification.

is in $|\mathbf{0}\rangle=(1,0,0 \ldots, 0,0)^{T}$ state. Therefore, we need to construct a $U_{f}$ which marks the nonzero eigenvalues and does nothing to $|\mathbf{0}\rangle$. This can be done by using a vector $|\mathbf{f}\rangle$ in the standard basis which has the same non-zero coefficients for the all basis states except the first one

$$
U_{f}=I-2|\mathbf{f}\rangle\langle\mathbf{f}|, \text { with }|\mathbf{f}\rangle=\frac{1}{\mu}\left(\begin{array}{c}
0 \\
1 \\
1 \\
\vdots \\
1
\end{array}\right) .
$$

Here, $\mu$ is a normalization constant equal to $\frac{1}{\sqrt{M-1}}$. $U_{f}$ does nothing when the first register in $|\mathbf{0}\rangle$ state; however, it does not only flip the signs but also changes the amplitudes of the other states. Then, $U_{\psi}$ is applied for the amplification of the marked amplitudes. The iterative application of $U_{\psi} U_{f}$ results in a quantum state where the amplitude of $|\mathbf{0}\rangle$ becomes almost zero and the amplitudes of the other states become almost equal. At this point, the second register holds $Q Q^{T}|\mathbf{x}\rangle$ which is the expected output from the neural network. This is explained in more mathematical terms below.

\subsection{Details of the Algorithm}

Here, we assume that $U=e^{l W t}$ is given: Later, in Section 3.4, we shall also discuss how $U$ may be obtained as a quantum circuit from a given $W$ matrix.

Figure 2 shows the algorithm as a quantum circuit where the dashed lines indicate an iteration in the amplitude amplification. At the beginning, $U_{P E A}$ is applied to the initial state $|\mathbf{0}\rangle|\mathbf{0}\rangle$. Note that $U_{P E A}$ includes also an input preparation circuit, $U_{\text {input }}$, bringing the second register from $|\mathbf{0}\rangle$ state to the input $|\mathbf{x}\rangle$. $U_{P E A}$ generates a superposition of the eigenvalues and associated eigenvectors, respectively, on the first and the second registers with the amplitudes defined by the overlap of the eigenvector and the input $|\mathbf{x}\rangle$

$$
|\psi\rangle=U_{P E A}|\mathbf{0}\rangle|\mathbf{0}\rangle=\sum_{j=0}^{N-1} \alpha_{j}\left|\lambda_{j}\right\rangle\left|\varphi_{j}\right\rangle,
$$

where $\alpha_{j}=\left\langle\varphi_{j}|| \mathbf{x}\right\rangle$. 
In the second part, the operator $G=U_{\psi} U_{f}$ is applied to $|\psi\rangle$ iteratively until $Q Q^{T}|\mathbf{x}\rangle$ can be obtained on the second register. The action of $U_{f}$ applied to $|\psi\rangle$ is as follows

$$
\begin{aligned}
\left|\psi_{1}\right\rangle & =U_{f}|\psi\rangle=(I-2|\mathbf{f}\rangle\langle\mathbf{f}|) \sum_{j=0}^{N-1} \alpha_{j}\left|\lambda_{j}\right\rangle\left|\varphi_{j}\right\rangle \\
& =|\psi\rangle-2 \mu|\mathbf{f}\rangle|\bar{\varphi}\rangle .
\end{aligned}
$$

Here, assuming the first $k$ number of eigenvalues are zero, the unnormalized state $|\bar{\varphi}\rangle$ is defined as

$$
|\bar{\varphi}\rangle=\sum_{j=k}^{N-1} \alpha_{j}\left|\varphi_{j}\right\rangle .
$$

It is easy to see that $|\bar{\varphi}\rangle=Q Q^{T}|\mathbf{x}\rangle$, which is our target output. When $U_{\psi}$ is applied to the output in Eq. [16), we simply change the amplitudes of $|\psi\rangle$

$$
\begin{aligned}
U_{\psi}\left|\psi_{1}\right\rangle & =U_{\psi}(|\psi\rangle-2 \mu|\mathbf{f}\rangle|\bar{\varphi}\rangle) \\
& =(I-2|\psi\rangle\langle\psi|)|\psi\rangle-2 \mu(I-2|\psi\rangle\langle\psi|)|\mathbf{f}\rangle|\bar{\varphi}\rangle \\
& =-|\psi\rangle-2 \mu\left(I-2|\psi\rangle \sum_{j=0}^{N-1} \alpha_{j}\left\langle\varphi_{j}\left|\left\langle\lambda_{j}\right|\right) \mid \mathbf{f}\right\rangle|\bar{\varphi}\rangle\right. \\
& =-|\psi\rangle-2 \mu|\mathbf{f}\rangle|\bar{\varphi}\rangle+4 \mu^{2} P_{f}|\psi\rangle \\
& =\left(4 \mu^{2} P_{f}-1\right)|\psi\rangle-2 \mu|\mathbf{f}\rangle|\bar{\varphi}\rangle .
\end{aligned}
$$

Here, $P_{f}$ is the initial success probability and equal to $\sum_{j=k}^{N-1} \alpha_{j}^{2}$. The repetitive applications of $G$ only changes the amplitudes of $|\psi\rangle$ and $|\mathbf{f}\rangle|\bar{\varphi}\rangle$ : e.g.,

$$
\begin{array}{r}
G^{2}|\psi\rangle=\left(c^{2}-3 c+1\right)|\psi\rangle-(c-2) 2 \mu|\mathbf{f}\rangle|\bar{\varphi}\rangle \\
G^{3}|\psi\rangle=\left(c^{3}-5 c^{2}+6 c-1\right)|\psi\rangle-\left(c^{2}-4 c+3\right) 2 \mu|\mathbf{f}\rangle|\bar{\varphi}\rangle
\end{array}
$$

where $c=\left(4 \mu^{2} P_{f}-1\right)$. The normalized probability of $(2 \mu|\mathbf{f}\rangle|\varphi\rangle)$ is presented in Figure 3 by using different values for $c$ (The amplitudes of $|\psi\rangle$ and $(2 \mu|\mathbf{f}\rangle)$ are normalized.). The amplitude of $|\psi\rangle$ through the iterations of the amplitude amplification oscillates with a frequency depending on the overlaps of the input with the eigenvectors. When the amplitude of $|\psi\rangle$ becomes close to zero, the second register in the remaining part $|\mathbf{f}\rangle|\bar{\varphi}\rangle$ is exactly $Q Q^{T}|\mathbf{x}\rangle$ and the first register is equal to $|\mathbf{f}\rangle$.

Figure 4 represents the iterations of the algorithm for a random $2^{7} \times 2^{7}$ matrix with $2^{7} / 2$ number of zero eigenvalues and a random input $|\mathbf{x}\rangle$. In each subfigure, we have used different numbers of qubits for the first register to see the effect on the results. The bar graphs in the subfigures shows the probability change for each state $|\mathbf{j}\rangle, j=0 \ldots 1$, of the first qubit (A different color tone indicates a different state.). When the probability for $|\mathbf{0}\rangle$ becomes close

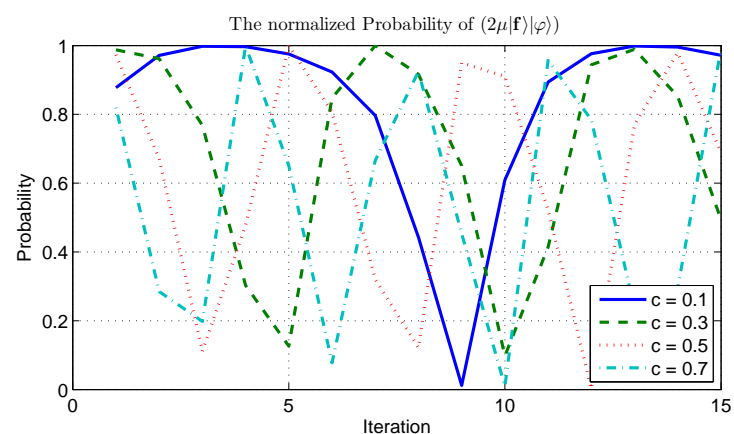

Figure 3: The normalized probability of $(2 \mu|\mathbf{f}\rangle|\varphi\rangle)$ through the iterations for different values of $c$.

to zero, the probabilities for the rest of the states become equal and so the total sum of these probabilities as shown in the bottom figure of each subfigure becomes almost one. At that point, the fidelity found by $\left|\left\langle\operatorname{reg}_{2}\left|Q Q^{T}\right| \mathbf{x}\right\rangle\right|$ also comes closer to one.

\subsection{Number of Iterations}

Through the iterations, while the probability for $|\mathbf{0}\rangle$ state goes to zero, the probabilities for the rest of the states become almost equal. This indicates that the individual states of each qubit turn into the equal superposition state. Therefore, if the state of a qubit in the first register is in the almost equal superposition state, then the success probability is very likely to be in its maximum level. In the Hadamard basis, $|0\rangle$ and $|1\rangle$ are represented in the equal superposition states as follows

$$
|0\rangle=\frac{|\mathbf{0}\rangle+|\mathbf{1}\rangle}{\sqrt{2}} \text { and }|1\rangle=\frac{|\mathbf{0}\rangle-|\mathbf{1}\rangle}{\sqrt{2}} .
$$

Therefore, using the Hadamard basis, if the probability of measuring $|0\rangle$ is close to one, in other words, if $|1\rangle$ is not seen in the measurement, then the second register likely holds $Q Q^{T}|\mathbf{x}\rangle$ with a maximum possible fidelity. Figure 5 shows the comparisons of the individual qubit probabilities (i.e., the probability to see a qubit in the first register in $|0\rangle$ in the Hadamard basis.) with the total probability observed in Figure $4 \mathrm{f}$ for the random case: As seen in the figure, the individual probabilities exhibit the same behavior as the total probability.

Generally, obtaining a possible probability density of an unknown quantum state is a difficult task. However, since we are dealing with only a single qubit and do not require the exact density, this can be done efficiently. For instance, if $|0\rangle$ is seen $n$ number of times in ten measurements, then the success probability is expected to be $n / 10$. Here, the number of measurements obviously determines the precision in the obtained probability which may also affect the fidelity. 


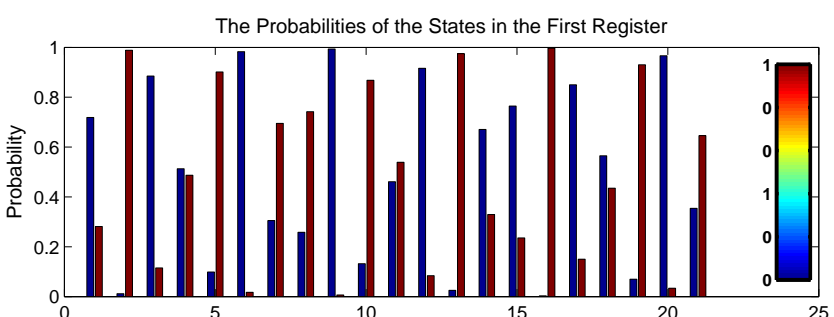

Iteration

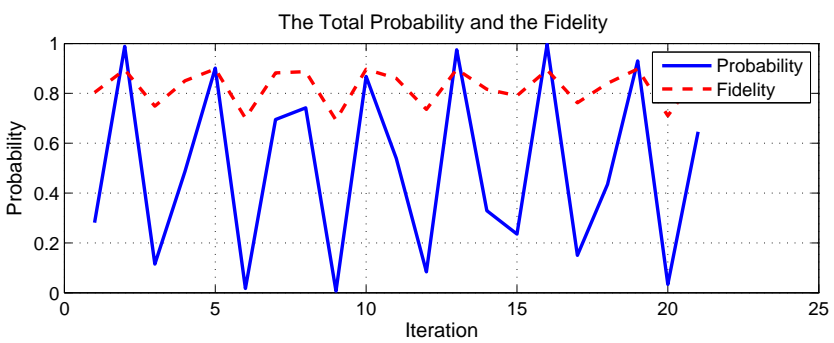

(a) $m$ is 1 .
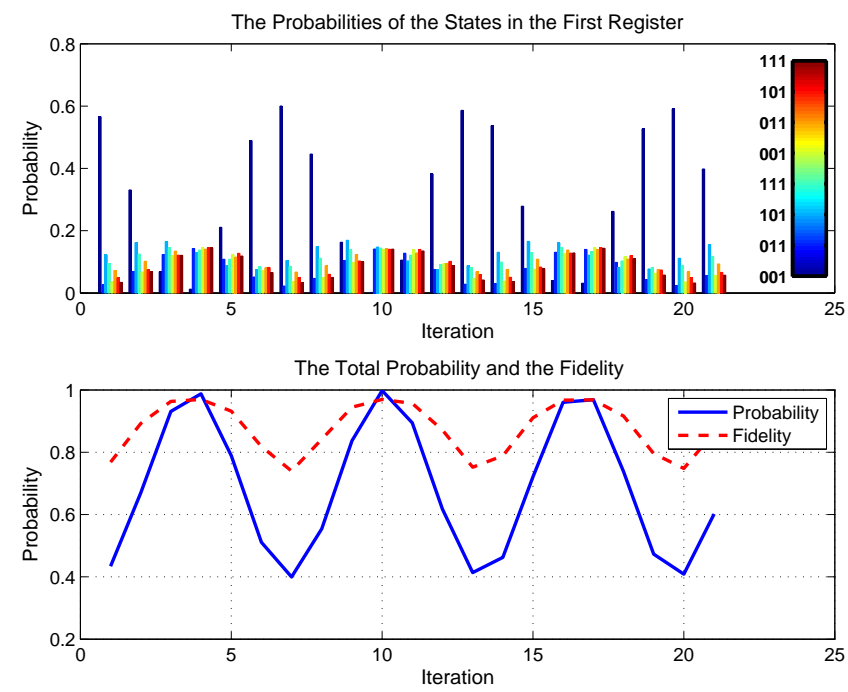

(c) $m$ is 3 .
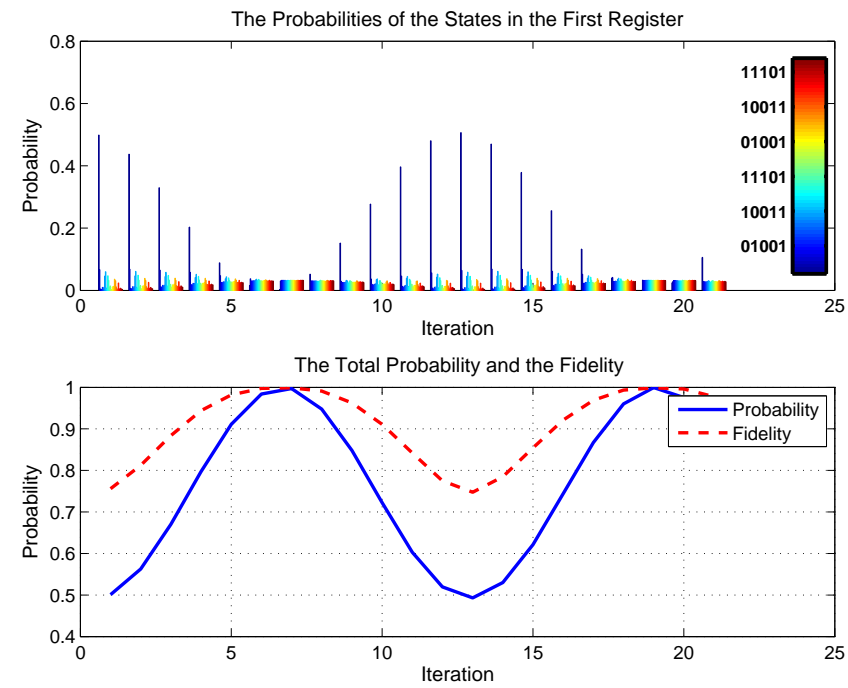

(e) $m$ is 5 .
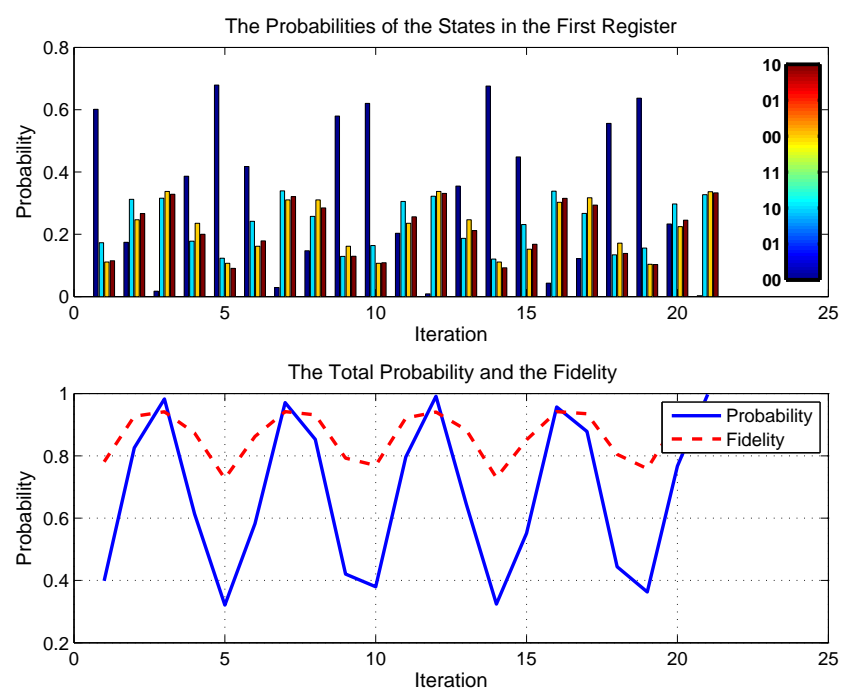

(b) $m$ is 2 .
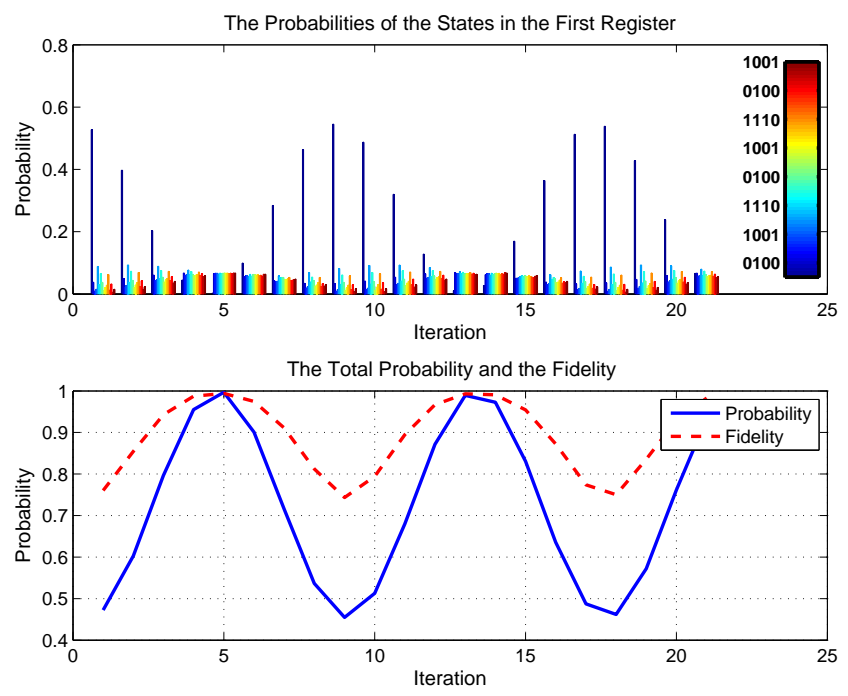

(d) $m$ is 4 .
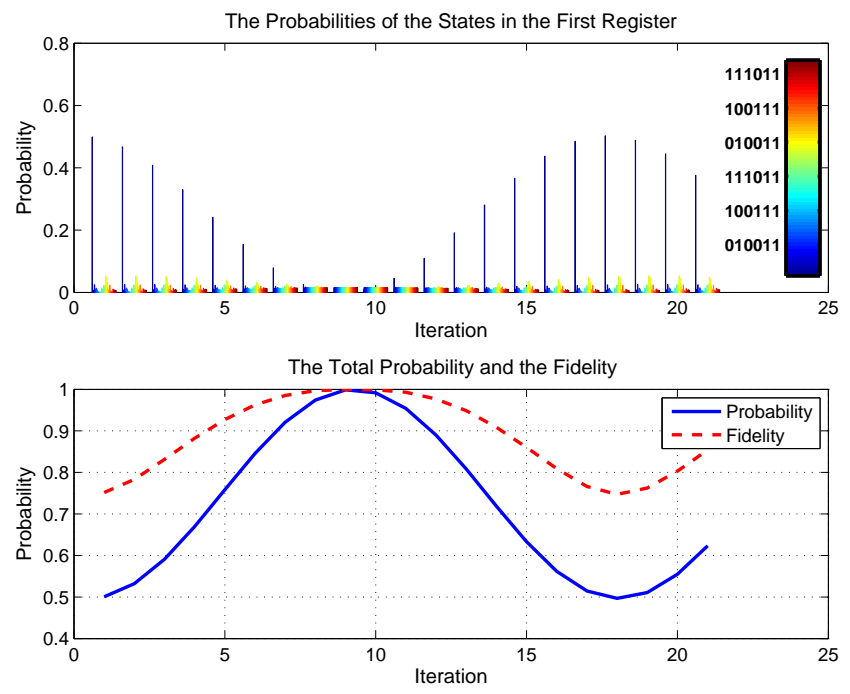

(f) $m$ is 6.

Figure 4: The probability changes in the iteration of the amplitude amplification for a random $2^{7} \times 2^{7}$ matrix with $2^{7} / 2$ number of zero eigenvalues and a random input $|\mathbf{x}\rangle$ (MATLAB code for the random generation is given in Appendix). In each subfigure, we have used different numbers of qubits, $m$, for the first register to see the effect on the results. The bar graphs in the subfigures shows the probability change for each state $|\mathbf{j}\rangle, j=0 \ldots 1$, of the first qubit. For each state, a different color tone is used. 


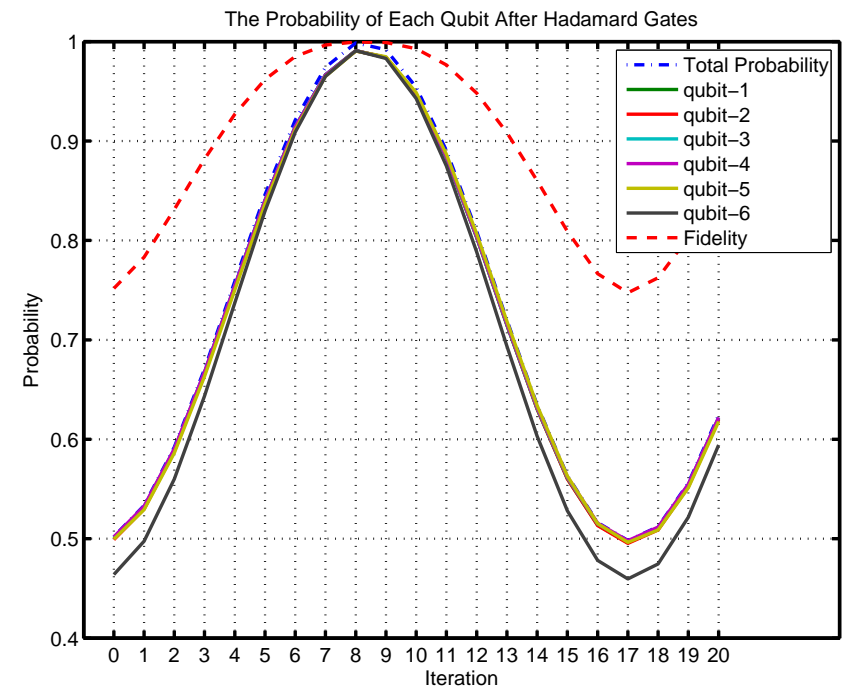

Figure 5: The probability to see a qubit in the first register in $|0\rangle$ state after applying a Hadamard gate to the qubit and its comparison with the total probability and the fidelity given in Figure 4f Note that the above separate curve is the fidelity. Since there are only small differences between the probabilities on the individual qubits and the total probability, the curves for the probabilities mostly overlap.

\subsection{Error-Precision (Number of Qubits in $\left.\left|\operatorname{reg}_{1}\right\rangle\right)$}

The number of qubits, $m$, in the first register should be sufficient to distinguish very small nonzero eigenvalues from the ones which are zero. In our numerical random experiments, we have observed that choosing only six or five qubits are enough to get very high fidelity while not requiring a high number of iterations. The impact of the number of qubits on the fidelity and the probability is shown in Figure 4 in which each sub-figure is drawn by using different register sizes for the same random case. As seen in the figure, the number of qubits also affects the required number of iterations: e.g., while for $m=3$, the highest fidelity and probability are seen at the fourth iteration; for $m=6$, it happens around the ninth iteration.

\subsection{Circuilt Implementation of $W$}

The circuit implementation of $W$ requires forming a quantum circuit representing the time evolution of $W$ : i.e., $U=e^{i 2 \pi W t}$. When $W$ is a sparse matrix, the circuit can be formed by following the method in [38]. However, when it is not sparse but in the following form $W=\sum_{j} \mathbf{x}_{\mathbf{j}} \mathbf{x}_{\mathbf{j}}^{T}$, then the exponential becomes equal to

$$
U=e^{i 2 \pi W t}=e^{i 2 \pi t \sum_{j} \mathbf{x}_{\mathbf{j}} \mathbf{x}_{\mathbf{j}}^{T}} .
$$

To approximate the above exponential, we apply the Trotter-Suzuki formula [39-42] to decompose Eq. (21) into the terms $U_{j}=e^{i 2 \pi \mathbf{x}_{\mathrm{j}} \mathbf{x}_{\mathbf{j}}{ }^{T}}=U_{\mathbf{x}_{\mathbf{j}}} \bar{I} U_{\mathbf{x}_{\mathbf{j}}}^{\dagger}$, where $\bar{I}$ is a kind of identity matrix with the first element set to $e^{\imath 2 \pi t}$, and $U_{\mathbf{x}_{\mathbf{j}}}$ is a unitary matrix with the first row and column equal to $\mathbf{x}_{\mathbf{j}}$. For instance, if the second order TrotterSuzuki decomposition is applied to Eq. (21) (note that the order of the decomposition impacts the accuracy of the approximation), the following is obtained

$$
e^{i 2 \pi t \sum_{j=1}^{\kappa} \mathbf{x}_{\mathbf{j}} \mathbf{x}_{\mathbf{j}}^{T}} \approx U_{j}\left(e^{i 2 \pi \frac{t}{2} \sum_{j=2}^{\kappa} \mathbf{x}_{\mathbf{j}} \mathbf{x}_{\mathbf{j}}^{T}}\right) U_{j}
$$

Then, the same decomposition is applied to the term $e^{i 2 \pi t / 2 \sum_{j=2}^{k} \mathbf{x}_{\mathbf{j}} \mathbf{x}_{\mathbf{j}}^{T}}$ in the above equation. This recursive decomposition yields an approximation composed of $(4 \kappa)$ number of $U_{\mathbf{x}_{\mathbf{j}}}$ matrices. Any $U_{\mathbf{x}_{\mathbf{j}}}$ can be implemented as a Householder matrix by using $O\left(2^{n}\right)$ quantum operations which is linear in the size of $\mathbf{x}_{\mathbf{j}}[43-46]$.

\subsection{Obtaining a solution from the output}

Generally, the amplitudes of the output vector (the final state of the second register) encodes the information needed for the solution of the considered problem. Since obtaining the full density of a quantum state is known to be very inefficient for larger systems, one needs to drive efficient measurement schemes specific to the problem. For instance, for some problems, comparisons of the peak values instead of the whole vectors may be enough to gauge a conclusion: In this case, since a possible outcome in a measurement would be the one with an amplitude likely to be greater than most of the states in magnitude, the peak values can be obtained efficiently. However, this alone may not be enough for some applications.

Moreover, in some applications such as the spectral clustering problem, a superposition of vectors that are forming a solution space for the problem can be used as an input state. In that case, the measurement of the output in the solution space yields the solution for the problem. This method can be used efficiently (polynomial time complexity in the number of qubits) when the vectors describing the solution space are tensor product of Pauli matrices.

\section{Complexity Analysis}

The computational complexity of a quantum algorithm is assessed by the total number of single gates and two qubit controlled NOT (CNOT) gates in the quantum circuit implementing the algorithm. We derive the computational complexity of the whole method by finding the complexities of $U_{f}$ on the first register with $m$ number of qubits and $U_{\psi}$ on the second register with $n$ number of qubits. We shall use $M=2^{m}$ and $N=2^{n}$ to describe the sizes of the operators on the registers. 


\subsection{The complexity of $U_{f}$}

It is known that the number of quantum gates to implement a Householder matrix is bounded by the size of the matrix [43-46]. Therefore, the circuit for $U_{f}$ requires $O(M)$ CNOT gates since it is a Householder transformation formed by the vector $|\mathbf{f}\rangle$ of size $M$.

\subsection{The complexity of $U_{\psi}$}

The operator $U_{\psi}$ is equal to $U_{P E A} U_{0} U_{P E A}^{\dagger}$ in which the total complexity will be typically governed by the complexity of $U_{P E A}$. $U_{P E A}$ involves the Fourier transforms, input preparation circuit, and the controlled $U=e^{\imath t W}$ with different $t$ values:

- The circuits for the quantum Fourier transform and its inverse are well known [36] and can be implemented on the first register in $O\left(\mathrm{~m}^{2}\right)$.

- The input preparation circuit on the second register, $U_{\text {input }}$, can be implemented again as a Householder transformation by using $O(N)$ number of quantum gates. It can be also designed by following Section III.B. of [47]: In that case, for every two vector elements, a controlled rotation gate is used to construct $U_{\text {input }}$ with the initial row equal to $\mathbf{x}$; thus, $U_{\text {input }}|\mathbf{0}\rangle=|\mathbf{x}\rangle$.

- The circuit complexity of $U=e^{\imath t W}$ is highly related to the structure of $W$. When $W$ of order $N$ is sparse enough: i.e., the number of nonzero entries is bounded by some polynomial of the number of qubits, poly(n); then $W$ can be simulated by using only $O(\operatorname{poly}(n))$ number of quantum gates [38, 48, 49]. However, when $W$ is not sparse but equal to $\sum \mathbf{x}_{\mathbf{i}} \mathbf{x}_{\mathbf{i}}{ }^{T}$, then as shown in Section 3.4, we use Trotter-Suzuki formula which yields a product of $(4 \kappa)$ number of $U_{\mathbf{x}_{\mathbf{j}}}$ matrices with $1 \leq j \leq \kappa$. Since $U_{\mathbf{x}_{\mathbf{j}}}$ can be implemented as a Householder transformation by using $O(N)$ quantum gates, $U$ requires $O(\kappa N)$ quantum gates.

If we combine all the above terms, the total complexity can be concluded as

$$
O(\kappa N+M)
$$

This is linear in system-size, however, exponential in the number of qubits involved in either one of the registers. In comparison, any classical method applied to obtain $Q Q^{T} \mathbf{x}$ at least requires $O\left(N^{2}\right)$ time complexity because of the matrix vector multiplication. Therefore, the quantum model presented here may provide a quadratic speedup over the classical methods for some applications. When the weight matrix is sparse or the data is given as a quantum states, it can be implemented in $O(\operatorname{poly}(n))$. Therefore, the whole complexity becomes linear in the number of qubits, which may provide an exponential speedup over the classical algorithms. However, when the weight matrix is not sparse, the complexity becomes exponential in the number of qubits. The current experimental research by big companies such as Google and IBM aims to build 50 qubit operational quantum computers [50]. Because of the limitations of the current quantum computer technology, when the required number of qubits goes beyond 50 , the applications of the algorithm becomes infeasible.

\section{An IIlustrative Example}

Here, we give a simple example to show how the algorithm works: Let us assume, we have given weights represented by the columns of the following matrix [51]

$$
X=\frac{1}{10} \times\left(\begin{array}{cc}
-1 & +1 \\
-1 & -1 \\
+1 & -1 \\
-1 & +1
\end{array}\right)
$$

where we scale the vectors by $\frac{1}{10}$ so as to make sure that the eigenvalues of $W$ are less than one. To validate the simulation results, first, $W_{[\infty]}$ is classically computed by following the singular value decomposition of $X$

$Q \Phi P^{T}=\left(\begin{array}{cc}+.5774 & 0 \\ 0 & 1 \\ -.5774 & 0 \\ +.5774 & 0\end{array}\right)\left(\begin{array}{cc}.2495 & 0 \\ 0 & .14142\end{array}\right)\left(\begin{array}{cc}-.7071 & +.7071 \\ -.7071 & -.7071\end{array}\right)$

Therefore,

$$
W_{[\infty]}=Q Q^{T}=\left(\begin{array}{cccc}
+.333 & 0 & -.333 & +.333 \\
0 & 1 & 0 & 0 \\
-.333 & 0 & +.333 & -.333 \\
+.333 & 0 & -.333 & +.333
\end{array}\right)
$$

We use the following Trotter-Suzuki formula [39-42] to compute the exponential of $W=X X^{\prime}$

$$
U=e^{l 2 \pi W} \approx e^{l \pi \mathbf{x}_{2} \mathbf{x}_{2}^{\mathrm{T}}} e^{l 2 \pi \mathbf{x}_{1} \mathbf{x}_{1}^{\mathbf{T}}} e^{l \pi \mathbf{x}_{2} \mathbf{x}_{2}^{\mathbf{T}}}
$$

In the simulation for a random input $|\mathbf{x}\rangle$, the comparison of $W_{[\infty]}|\mathbf{x}\rangle=Q Q^{T}|\mathbf{x}\rangle$ with the output of the second register in the quantum model yields the fidelity. For two different random inputs, the simulation results in each iteration are shown in Figure 6a and Figure $6 \mathrm{~b}$ for $|\mathbf{x}\rangle=(.3517 .3058 .6136 .6374)^{T}$ and $|\mathbf{x}\rangle=(.7730 .1919 .1404 .5881)^{T}$, respectively. 

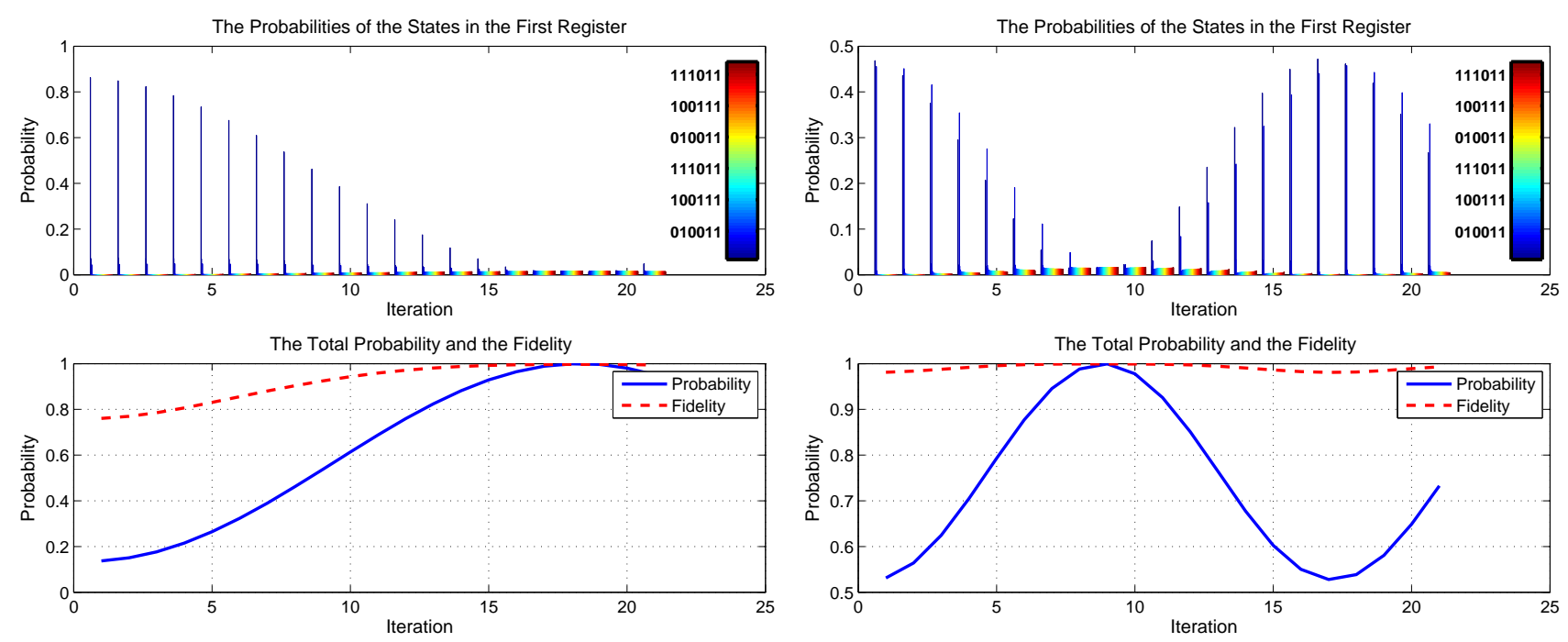

(a) For the generated random input $|\mathbf{x}\rangle=(.3517 .3058 .6136 .6374)^{T}$.(b) For the generated random input $|\mathbf{x}\rangle=(.7730 .1919 .1404 .5881)^{T}$.

Figure 6: The simulation results of the quantum model for the example in Section 5 with two different input vectors.

\section{Conclusion}

The weight matrix of the networks based on the WidrowHoff learning rule converges to $Q Q^{T}$, where $Q$ represents the eigenvectors of the matrix corresponding to the nonzero eigenvalues. Here, we applied the quantum principal component analysis method described in [35] to artificial neural networks using the Widrow-Hoff learning rule and showed that one can implement an equivalent quantum circuit which produces the output $Q Q^{T} \mathbf{x}$ for a given input $\mathbf{x}$ in linear time. We also discussed the implementation details by using random cases, analyzed the computation complexity based on the number of quantum gates and presented a simple numerical example. The model is general and requires only linear time computational complexity in the size of the weight matrix.

\section{Appendix: MATLAB Code for the Random Matrix}

The random matrix used in the numerical example is generated by the following MATLAB code snippet:

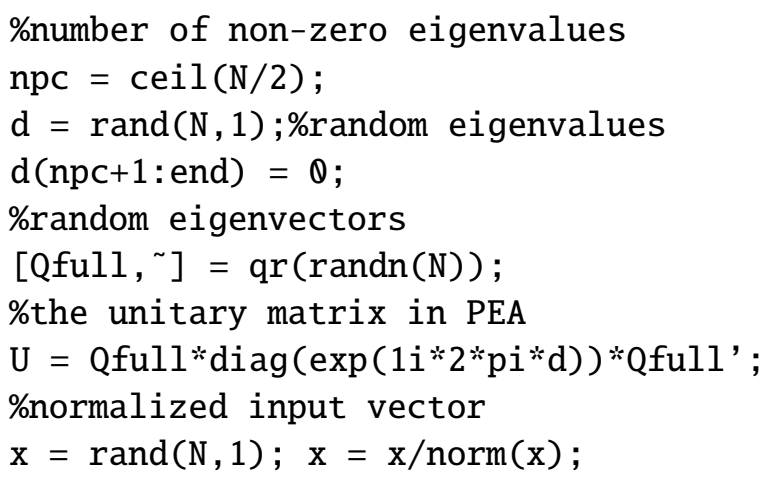

$\mathrm{npc}=\operatorname{ceil}(\mathrm{N} / 2)$;

$\mathrm{d}=\operatorname{rand}(\mathrm{N}, 1) ; \%$ random eigenvalues

$\mathrm{d}(\mathrm{npc}+1:$ end $)=0$

\%random eigenvectors

$[Q$ full,$\sim]=\operatorname{qr}(\operatorname{randn}(\mathrm{N}))$;

\%the unitary matrix in PEA

$\mathrm{U}=\mathrm{Qfull} * \operatorname{diag}(\exp (1 \mathrm{i} * 2 * \mathrm{pi} * \mathrm{~d})){ }^{*} \mathrm{Qfull}{ }^{\prime}$;

$\mathrm{x}=\operatorname{rand}(\mathrm{N}, 1) ; \mathrm{x}=\mathrm{x} / \operatorname{norm}(\mathrm{x})$;

\section{References}

[1] Haykin SO. Neural Networks and Learning Machines, 3rd edition. Upper Saddle River, New Jersey: Pearson, 2008.

[2] Abdi H. Linear algebra for neural networks. In: International Encyclopedia of the Social and Behavioral Sciences. Baltes PB (editor), Oxford: Pergamon, 2001, pp. 8864-8868. doi : 10.1016/B0-08043076-7/00609-4

[3] Abdi H, Valentin D, Edelman B, O'Toole AJ. More about the difference between men and women: evidence from linear neural networks and the principalcomponent approach. Perception 1995; 24(5): 539562. doi : $10.1068 / \mathrm{p} 240539$

[4] Morris RGM. D. O. Hebb: The Organization of Behavior, Wiley: New York; 1949. Brain Research Bulletin 1999; 50(5): 437. doi:10.1016/S03619230(99)00182-3

[5] Widrow B, Hoff ME. Adaptive switching circuits. In: Neurocomputing: Foundations of Research. Anderson JA, Rosenfeld E (editors), Cambridge, Massachusetts: MIT Press, 1988, pp. 123-134.

[6] Abdi H, Valentin D, Edelman B, O'Toole AJ. A Widrow-Hoff learning rule for a generalization of the linear auto-associator. Journal of Mathematical Psychology 1996; 40(2): 175-182. doi:10.1006/ jmps.1996.0017

[7] Shor PW. Algorithms for quantum computation: discrete logarithms and factoring. Proceedings of 
the 35th Annual Symposium on Foundations of Computer Science, November, 20-22, pp. 124-134. doi : 10.1109/sfcs. 1994.365700

[8] Grover LK. A fast quantum mechanical algorithm for database search. Proceedings of the 28th annual ACM symposium on Theory of Computing, Philadelphia, Pennsylvania, May 22-24, 1996, Association for Computing Machinery, pp. 212-219. doi: 10.1145/237814.237866

[9] Neven H, Denchev VS, Rose G, Macready WG. Training a binary classifier with the quantum adiabatic algorithm. 2008. arXiv:0811.0416

[10] Neven H, Denchev VS, Rose G, Macready WG. Training a large scale classifier with the quantum adiabatic algorithm. 2009. arXiv:0912.0779

[11] Aïmeur E, Brassard G, Gambs S. Quantum speedup for unsupervised learning. Machine Learning 2013; 90(2): 261-287. doi:10.1007/s10994012-5316-5

[12] Lloyd S, Garnerone S, Zanardi P. Quantum algorithms for topological and geometric analysis of data. Nature Communications 2016; 7(10138. arXiv:1408.3106, doi : $10.1038 /$ ncomms 10138

[13] Rebentrost P, Mohseni M, Lloyd S. Quantum support vector machine for big data classification. Physical Review Letters 2014; 113(13): 130503. arXiv:1307.0471, doi : 10.1103/PhysRevLett. 113.130503

[14] Wittek P. Quantum Machine Learning: What Quantum Computing Means to Data Mining. Amsterdam: Academic Press, 2014.

[15] Schuld M, Sinayskiy I, Petruccione F. Quantum computing for pattern classification. Proceedings of the Pacific Rim International Conference on Artificial Intelligence 2014: Trends in Artificial Intelligence, Cham, Pham D-N, Park S-B (editors), Springer, pp. 208-220. arXiv: 1412.3646, doi : 10.1007/978-3-319-13560-1_17

[16] Lloyd S, Mohseni M, Rebentrost P. Quantum principal component analysis. Nature Physics 2014; 10(9): 631-633. arXiv:1307.0401, doi:10. $1038 /$ nphys 3029

[17] Manju A, Nigam MJ. Applications of quantum inspired computational intelligence: a survey. $A r$ - tificial Intelligence Review 2014; 42(1): 79-156. doi : 10.1007/s10462-012-9330-6

[18] da Silva AJ, Ludermir TB, de Oliveira WR. Quantum perceptron over a field and neural network architecture selection in a quantum computer. Neural Networks 2016; 76: 55-64. arXiv: 1602.00709, doi:10.1016/j.neunet.2016.01.002

[19] Zhou R, Wang H, Wu Q, Shi Y. Quantum associative neural network with nonlinear search algorithm. International Journal of Theoretical Physics 2012; 51(3): 705-723. doi:10.1007/s10773011-0950-4

[20] Gupta S, Zia RKP. Quantum neural networks. Journal of Computer and System Sciences 2001; 63(3): 355-383. doi: 10.1006/jcss.2001.1769

[21] Andrecut M, Ali MK. A quantum neural network model. International Journal of Modern Physics C 2002; 13(1): 75-88. doi:10.1142/ S0129183102002948

[22] Altaisky MV. Quantum neural network. 2001. arXiv:quant-ph/0107012

[23] Schuld M, Sinayskiy I, Petruccione F. Quantum walks on graphs representing the firing patterns of a quantum neural network. Physical Review A 2014; 89(3): 032333. arXiv: 1404.0159, doi: 10.1103/PhysRevA.89.032333

[24] Schuld M, Sinayskiy I, Petruccione F. The quest for a quantum neural network. Quantum Information Processing 2014; 13(11): 2567-2586. arXiv: 1408 . 7005, doi : 10.1007/s11128-014-0809-8

[25] Kouda N, Matsui N, Nishimura H, Peper F. Qubit neural network and its learning efficiency. Neural Computing and Applications 2005; 14(2): 114-121. doi : $10.1007 / \mathrm{s} 00521-004-0446-8$

[26] Li P, Xiao H, Shang F, Tong X, Li X, Cao M. A hybrid quantum-inspired neural networks with sequence inputs. Neurocomputing 2013; 117: 81-90. doi : 10.1016/j.neucom.2013.01.029

[27] Ventura D, Martinez T. A quantum associative memory based on Grover's algorithm. Proceedings of the Artificial Neural Nets and Genetic Algorithms, Vienna, Dobnikar A, Steele NC, Pearson DW, Albrecht RF (editors), Springer, pp. 22-27. doi : 10.1007/978-3-7091-6384-9_5 
[28] Kitaev AY. Quantum measurements and the Abelian Stabilizer Problem. Electronic Colloquium on Computational Complexity 1996; 3: TR96-003. arXiv: quant-ph/9511026, https://eccc.weizmann. ac.il/report/1996/003

[29] Harrow AW, Hassidim A, Lloyd S. Quantum algorithm for linear systems of equations. Physical Review Letters 2009; 103(15): 150502. arXiv: 0811.3171, doi:10.1103/PhysRevLett.103. 150502

[30] Grover LK. Quantum computers can search rapidly by using almost any transformation. Physical Review Letters 1998; 80(19): 4329-4332. arXiv:quant-ph/9712011, doi : 10.1103/PhysRevLett. 80.4329

[31] Mosca M. Quantum searching, counting and amplitude amplification by eigenvector analysis. In: MFCS'98 Workshop on Randomized Algorithms. 1998, pp. 90-100.

[32] Brassard G, Høyer P, Mosca M, Tapp A. Quantum amplitude amplification and estimation. In: Quantum Computation and Information. Lomonaco Jr. SJ, Brandt HE (editors), Contemporary Mathematics, vol. 305, Providence, Rhode Island: American Mathematical Society, 2002, pp. 53-74. arXiv: quant-ph/0005055, doi : 10.1090/conm/305

[33] Chen CL, Dong DY, Chen ZH. Quantum computation for action selection using reinforcement learning. International Journal of Quantum Information 2006; 4(6): 1071-1083. doi:10.1142/ S0219749906002419

[34] Dong D, Chen C, Li H, Tarn TJ. Quantum reinforcement learning. IEEE Transactions on Systems, Man, and Cybernetics, Part B (Cybernetics) 2008; 38(5): 1207-1220. doi:10.1109/tsmcb.2008.925743

[35] Daskin A. Obtaining a linear combination of the principal components of a matrix on quantum computers. Quantum Information Processing 2016; 15(10): 4013-4027. arXiv: 1512.02109, doi:10. $1007 / \mathrm{s} 11128-016-1388-7$

[36] Nielsen MA, Chuang IL. Quantum Computation and Quantum Information, 10th Anniversary edition. Cambridge: Cambridge University Press, 2010.

[37] Brassard G, Hoyer P. An exact quantum polynomialtime algorithm for Simon's problem. Proceedings of the Proceedings of the Fifth Israeli Symposium on Theory of Computing and Systems, June 1719, 1997, pp. 12-23. arXiv:quant-ph/9704027. doi:10.1109/istcs.1997.595153

[38] Berry DW, Ahokas G, Cleve R, Sanders BC. Efficient quantum algorithms for simulating sparse Hamiltonians. Communications in Mathematical Physics 2007; 270(2): 359-371. arXiv:quantph/0508139, doi: $10.1007 / \mathrm{s} 00220-006-0150$ $\mathrm{X}$

[39] Trotter HF. On the product of semi-groups of operators. Proceedings of the American Mathematical Society 1959; 10(4): 545-551. JSTOR:2033649, doi : 10.1090/S0002-9939-1959-0108732-6

[40] Suzuki M. Generalized Trotter's formula and systematic approximants of exponential operators and inner derivations with applications to many-body problems. Communications in Mathematical Physics 1976; 51(2): 183-190. doi:10.1007/bf01609348 https://projecteuclid.org/euclid.cmp/ 1103900351

[41] Hatano N, Suzuki M. Finding exponential product formulas of higher orders. In: Quantum Annealing and Other Optimization Methods. Das A, K. Chakrabarti B (editors), Lecture Notes in Physics, vol. 679, Berlin: Springer, 2005, pp. 37-68. arXiv: math-ph/0506007,doi:10.1007/11526216_2

[42] Poulin D, Hastings MB, Wecker D, Wiebe N, Doberty AC, Troyer M. The Trotter step size required for accurate quantum simulation of quantum Chemistry. Quantum Information and Computation 2015; 15(5-6): 361-384. arXiv: 1406.4920

[43] Ivanov PA, Kyoseva ES, Vitanov NV. Engineering of arbitrary $U(N)$ transformations by quantum Householder reflections. Physical Review A 2006; 74(2): 022323. arXiv:0708.2811, doi: 10.1103/PhysRevA.74.022323

[44] Urías J, Quiñones DA. Householder methods for quantum circuit design. Canadian Journal of Physics 2015; 94(2): 150-157. doi:10.1139/cjp2015-0490

[45] Ivanov PA, Vitanov NV. Synthesis of arbitrary unitary transformations of collective states of trapped ions by quantum Householder reflections. Physical Review A 2008; 77(1): 012335. doi : 10.1103/ PhysRevA.77.012335 
[46] Bullock SS, O'Leary DP, Brennen GK. Asymptotically optimal quantum circuits for $d$-level systems. Physical Review Letters 2005; 94(23): 230502. arXiv:quant-ph/0410116, doi:10. 1103/PhysRevLett. 94.230502

[47] Daskin A, Grama A, Kollias G, Kais S. Universal programmable quantum circuit schemes to emulate an operator. Journal of Chemical Physics 2012; 137(23): 234112. arXiv: 1204.3600, doi : $10.1063 / 1.4772185$

[48] Aharonov D, Ta-Shma A. Adiabatic quantum state generation and statistical zero knowledge. Proceedings of the 35th annual ACM symposium on Theory of Computing, San Diego, California, June 9-11, 2003, Association for Computing Machinery, pp. 20-29. arXiv:quant-ph/0301023, doi : 10.1145/780542.780546
[49] Childs AM, Kothari R. Simulating sparse Hamiltonians with star decompositions. In: Theory of Quantum Computation, Communication, and Cryptography. van Dam W, Kendon VM, Severini S (editors), Lecture Notes in Computer Science, vol. 6519, Berlin: Springer, 2011, pp. 94-103. arXiv: 1003 . 3683, doi:10.1007/978-3-642-18073-6_8

[50] Castelvecchi D. Quantum computers ready to leap out of the lab in 2017. Nature 2017; 541(7635): 9-10. doi: 10.1038/541009a

[51] Abdi H, Valentin D, Edelman B. Neural Networks. Quantitative Applications in the Social Sciences, vol. 124, Thousand Oaks, California: SAGE Publications, 1998. 